\section{PWE-170 A DEDICATED COLORECTAL CANCER GENETICS SERVICE IMPROVES ADHERENCE WITH MOLECULAR TESTING FOR LYNCH SYNDROME}

doi:10.1136/gutjnl-2013-304907.458

1."S Stevens, 'L Sergot, ' 1 F Turkes, '1 Smith, 'K Monahan. 'Family History of Bowel Cancer Clinic, West Middlesex University Hospital and Imperial College, NHS, London, UK

Introduction Lynch Syndrome (LS) accounts for 2-3\% of colorectal cancer (CRC); 1000 cases of CRC in the United Kingdom annually. It occurs as a result of mutations in DNA repair genes; limiting DNA repair and causing Microsatellite Instability (MSI). Previous studies have demonstrated that in current practise less than $10 \%$ of these cases are identified as LS due to a lack of appropriate testing with immunohistochemistry or MSI analysis. The international Revised Bethesda Criteria were devised in 2004 to help identify such cases; these criteria include all individuals diagnosed $<50$ years of age, those with synchronous or metachronous CRC or LS-related cancer, those with significant family history of CRC or a LS-related cancer, or individuals diagnosed $<60$ years of age with MSI-type histology.

Methods We identified all new cases of colorectal cancer over a 1 year period prior to and subsequent to the establishment of a dedicated 'Family History of Bowel Cancer Service'. Adherence to the Revised Bethesda Criteria was determined by examination of medical records and UK National Bowel Cancer Audit Programme (NBOCAP) data. Pathology reports were studied in patients aged under 60 years of age at diagnosis looking for features consistent with MSI-H histology. We used Chi-squared testing to calculate significance for binary variables. Results Over the two year period 198 cases of colorectal cancer were discussed at the CRC multidisciplinary meeting. 41 patients fulfilled the Revised Bethesda Criteria for screening for Lynch Syndrome; 12 individuals were diagnosed under the age of 50 years $(\sim 6 \%) ; 4$ patients were diagnosed under 60 years of age and had MSI-H type histology and 25 patients had a significant family history of CRC or a LS-related cancer. In the year prior to the introduction of the clinic, we identified 18 cases meeting the Revised Bethesda Criteria for screening for LS; however, only 1 patient had been tested $(5.6 \%)$. In contrast following the introduction of the clinic 19 of 23 identified cases (82.6\%) were tested. Chi-squared testing demonstrated clinical significance when comparing the screening prior to and subsequent to the introduction of the clinic, $p$ value $=9.7 \times 10-7($ Chi $=23.9956) .6$ of the screened cases demonstrated molecular features with MSI and abnormal Immunohistochemistry, and are undergoing further germline genetic testing.

Conclusion The establishment of a dedicated 'Family History of Bowel Cancer Service' resulted in a significant improvement in the screening for Lynch Syndrome in accordance with the Revised Bethesda Criteria, 2004. We would recommend that this service should be extended throughout the United Kingdom to help aid early diagnoses and improve long-term outcomes.

Disclosure of Interest None Declared.

\section{PWE-171 BONE MORPHOGENETIC PROTEIN (BMP) PATHWAY DYSREGULATION SUBVERTS ONCOGENE INDUCED SENESCENCE MECHANISMS IN THE SERRATED PATHWAY OF TUMOURIGENESIS}

doi:10.1136/gutjnl-2013-304907.459

1,2,*T Boitsova, ${ }^{1} \mathrm{H}$ Rafferty, ${ }^{1} \mathrm{H}$ Davis, ${ }^{1} \mathrm{C}$ Bardella, ${ }^{3} \mathrm{P}$ Gill, ${ }^{4} \mathrm{~J}$ East, ${ }^{1}$ I Tomlinson, ${ }^{3} \mathrm{R}$ Chetty, ${ }^{2} \mathrm{~A}$ Silver, ${ }^{1,4}$ S Leedham. 'Molecular and Population Genetics, Wellcome Trust Centre for Human Genetics, Oxford; ' ${ }^{2}$ Colorectal Cancer Genetics, Barts and the London Hospital Medical School, London; ${ }^{3}$ Histopathology; ${ }^{4}$ Translational Gastroenterology Unit, Oxford University Hospitals, Oxford, UK

Introduction The serrated colorectal carcinogenesis pathway is increasingly recognised as a distinct and important route to $\mathrm{CpG}$ island methylated phenotype (CIMP) carcinomas, yet comparatively little is known about the molecular pathogenesis of these lesions. The precursor lesions - hyperplastic and serrated polyps, are initiated by $B R A F$ or $K R A S$ mutations which initially induce proliferation but are followed by activation of oncogene-induced senescence (OIS) pathways resulting in telomere independent cell-cycle arrest. Subversion of OIS mechanisms is thus necessary for progression of serrated lesions. We assessed (epi)genetic mutation burden and morphogen expression in different colorectal polyps and investigated the effect of disrupted morphogen balance on OIS systems

Methods Colonoscopic samples of hyperplastic, serrated, conventional adenomas and neighbouring normal tissue were used. Individual crypt gene expression was measured, DNA extracted and (epi)mutation burden assessed. Epigenetic silencing of the BMP antagonist GREM2 was assessed in serrated polyps and a methylated cell-line. K-rasV12 transfection of mouse embryonic fibroblasts (MEF) was used to induce OIS and assess dynamic morphogen changes in senescing cells. shRNA knockdown of BMP4 in a cancer (HCT116) and primary immortalised (HCEC) cell-line was used to reverse the changes seen in human polyps.

Results Marked BMP component changes were seen in both serrated and traditional adenomas, with fewer changes in rectal hyperplastic polyps. Only serrated polyps showed aberrant gene methylation, including epigenetic transcriptional silencing of the $\mathrm{BMP}$ antagonist GREM2. Differential BMP ligand expression (BMP2 downregulated, $B M P 4$ upregulated) was noted in adenomas and confirmed with in situ hybridisation. KrasV12 OIS induction in MEF's caused the reversed expression pattern of BMP ligands to that seen in polyps, a change not seen in replicative senescence of the same cells. $B M P 4$ shRNA knockdown impeded anchorage-dependent growth of HCT116 cells and provoked a rapid cell senescence response in HCEC cells with growth arrest and up-regulation of both p21and $p 16$.

Conclusion We hypothesise that BMP ligands are involved in mediating (BMP2), or subverting (BMP4) oncogene-induced senescence, and that epigenetic silencing of specific BMP pathway constituents is selected early during serrated tumourigenesis. By demonstrating the mechanistic involvement of the BMP pathway in OIS we have identified an important early tumour promoting role of morphogen dysregulation which may inform future drug development.

Disclosure of Interest None Declared.

\section{PWE-172 MICRONUTRIENT-ANTIOXIDANT SUPPLEMENTATION HAS NO IMPACT ON GASTRIC ATROPHY IN ZAMBIAN ADULTS: A RANDOMISED CONTROLLED TRIAL}

doi:10.1136/gutjnl-2013-304907.460

1,"V Kayamba, ${ }^{2} \mathrm{M}$ S Chomba, ${ }^{3 P}$ Kelly. 'Dept Medicine, University Teaching Hospital, Lusaka, Zambia; '2Dept Medicine, UTH, Lusaka, Zambia; ${ }^{3 B l i z a r d ~ I n s t i t u t e, ~ B a r t s ~ \& ~ T h e ~}$ London, London, UK

Introduction With the increasing number of gastric cancer cases in young patients in Zambia, we set out to investigate the possibility of reversing gastric atrophy, a precancerous lesion, using micronutrient-antioxidant supplementation in a retrospective analysis of a published randomised controlled trial. We recently reported that low antioxidant levels were associated with gastric cancer in Zambia. In an earlier study, we had demonstrated that people with the HIV infection tend to have higher gastric $\mathrm{pH}$ than those without HIV.

Methods Archival samples from a randomised controlled trial, (Kelly et al Trans R Soc Trop Med Hyg. 2008; 102:194-9) were used in this study. These were collected from healthy volunteers in Misisi, a densely populated and impoverished township in Lusaka, and carefully stored at $-80^{\circ} \mathrm{C}$ in a secure laboratory at the University Teaching Hospital, the largest referral hospital in Zambia. In this controlled trial, 500 volunteers were randomly allocated to either a micronutrient-antioxidant supplementation or placebo. The 